\title{
Patchy reconnection in a Y-type current sheet
}

\author{
M. G. Linton ${ }^{1}$, C. R. DeVore ${ }^{1}$, and D. W. Longcope ${ }^{2}$ \\ ${ }^{1}$ Naval Research Laboratory, Washington, DC, U.S.A. \\ ${ }^{2}$ Montana State University, Bozeman, MT, U.S.A. \\ (Received November 21, 2007; Revised March 24, 2008; Accepted June 20, 2008; Online published May 29, 2009)
}

\begin{abstract}
We study the evolution of the magnetic field in a Y-type current sheet subject to a brief, localized magnetic reconnection event. The reconnection produces up- and down-flowing reconnected flux tubes which rapidly decelerate when they hit the Y-lines and underlying magnetic arcade loops at the ends of the current sheet. This localized reconnection outflow followed by a rapid deceleration reproduces the observed behavior of post-CME downflowing coronal voids. These simulations support the hypothesis that these observed coronal downflows are the retraction of magnetic fields reconnected in localized patches in the high corona.
\end{abstract}

Key words: Solar, corona, flare, reconnection.

\section{Introduction}

Reconnection is believed to be a key process allowing the excitation of solar flares and coronal mass ejections (CMEs). The reconnection releases significant magnetic energy, leading to solar flare heating, and changes magnetic topologies, allowing CME magnetic fields to erupt and escape the solar corona into interplanetary space. Observations of the flaring which occurs behind recently erupted CMEs show downflowing voids (see, e.g., McKenzie and Hudson, 1999; Gallagher et al., 2002; Innes et al., 2003; Asai et al., 2004; Sheeley et al., 2004) which push their way through the heated flare plasma in the high corona. These downflowing voids, observed by TRACE, Yohkoh SXT, and Hinode XRT, have been shown to be evacuated structures. They are therefore not cool, dense plasma blobs being pulled down by gravity, but rather appear to be evacuated loops of magnetic field being pulled down by the magnetic tension force (McKenzie and Hudson, 1999). The three dimensional (3D) structure of these voids breaks up the two dimensional (2D) symmetry of the flare current sheet and arcade, implying that the reconnection which creates these voids occurs in localized $3 \mathrm{D}$ patches rather than uniformly along the current sheet. The reconnected field from this patchy reconnection takes the shape of individual 3D flux tubes rather than extended 2D sheets of field. In this letter, we study whether the formation of magnetic loops high in the corona via a $3 \mathrm{D}$ patch of reconnection creates structures consistent with the morphology and dynamics of these coronal voids.

In Linton and Longcope (2006), we showed that the shapes and evolutions of such magnetic loops in a one dimensional (1D) Harris type current sheet are consistent with observations of these downflowing loops. The cross sections of the simulated magnetic loops form teardrop shapes,

Copyright (c) The Society of Geomagnetism and Earth, Planetary and Space Sciences (SGEPSS); The Seismological Society of Japan; The Volcanological Society of Japan; The Geodetic Society of Japan; The Japanese Society for Planetary Sciences; TERRAPUB. similar to that of the voids, while the $3 \mathrm{D}$ structure of the loops is similar to the structure of the coronal loops which appear below these voids, e.g., as seen by Sheeley et al. (2004).

However, there is a key aspect of the void dynamics which the initial 1D nature of the Harris current sheet cannot reproduce. This is the rapid deceleration of the voids once they reach the post-flare arcade loops in the low corona. Sheeley et al. (2004) have shown that the speeds of these voids through the high corona are relatively constant until they hit the coronal arcade, when they rapidly decelerate. As the Harris current sheet continues unchanged to the edge of the simulation, there is no arcade of loops at the base of such a current sheet with which the voids collide. To study this deceleration, we therefore now simulate this patchy reconnection in a Y-type current sheet (Green, 1965). The Y-type current sheet terminates at a set of magnetic arcade loops, as shown in Figs. 1(a) and 2(a). The intersections of the current sheet and the outermost of these arcade loops forms the two Y-lines, at $z= \pm L$ in Fig. 1(a). These arcades make the current sheet more representative of a post-CME coronal current sheet with underlying arcade fields. Note that these Y-lines are not Y-type nulls, as there is a uniform guide field in both the current sheet and the arcade, so the field strength does not go to zero at the Ylines, even though the reconnection component of the field does go to zero here.

We study the effect of a localized reconnection event in this current sheet, focusing on the form of the reconnected field, and on whether it decelerates once it hits the Y-line and the coronal arcade below it. The current sheet configuration and the simulation setup are discussed in Section 2, the results are described in Section 3, and our conclusions are summarized in Section 4.

\section{Simulations}

The simulations were performed using the magnetohydrodynamic (MHD) code ARMS (Adaptively Refined 


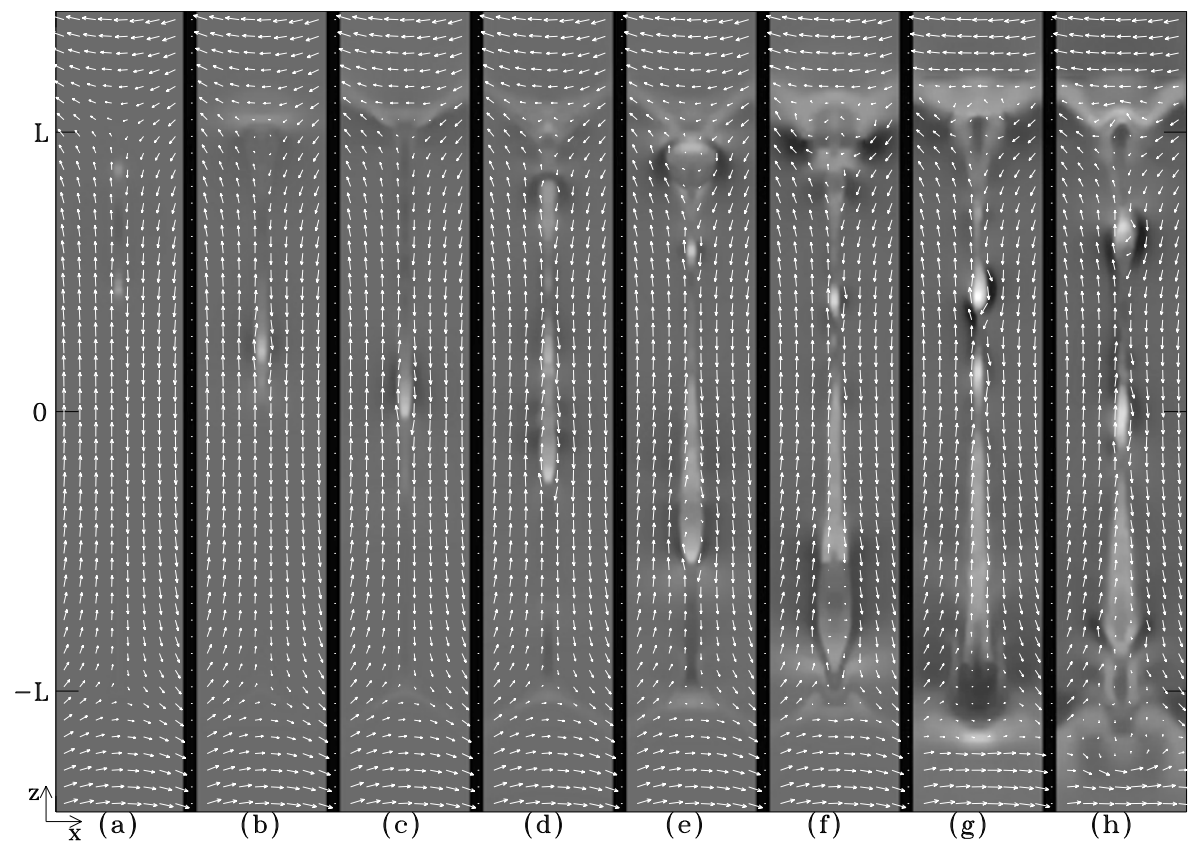

Fig. 1. Magnetic field of the Y-type current sheet in the $y=0$ plane, with a magnetic reconnection patch imposed at $z=2 L / 3$ for a time $t v_{A 0} / L=0.6$. The vectors show the magnetic field in the plane, while the greyscale shows the guide field component, with white representing maximum positive field. The panels show the simulation at times $t v_{A 0} / L=[0.2,0.7,1.1,1.6,2.1,2.6,3.1,3.5]$. The $x$ boundaries of each panel shown are at $x= \pm 3 L / 4 \pi$

MHD Solver) on the Cray XD1 supercomputer at the Naval Research Laboratory. The code was used to solve the resistive MHD equations. See Welsch et al. (2005) for a discussion of the code, and a presentation of the equivalent ideal MHD equations. We include an explicit resistivity $\eta$ in the induction equation, $\partial \mathbf{B} / \partial t=\nabla \times(\mathbf{v} \times \mathbf{B}-\eta \nabla \times \mathbf{B})$, and an ohmic heating term in the energy equation.

The Y-type magnetic field (e.g., Priest and Forbes, 2000) is

$$
B_{x}+i B_{z}=-B_{0} \sqrt{\omega^{2} / L^{2}-1},
$$

where $i$ is the positive value of $\sqrt{-1}, \omega \equiv z+i x$, and $L$ is the current sheet half-length. The guide field is uniform at $B_{y}=B_{0} / \pi$. Here $B_{z}(z=0, x \sim 0)=B_{0}=44$ in units where the pressure is $p_{0}=20 / 3$. Due to the guide field, the magnetic fieldlines on either side of the current sheet form a half angle $\zeta=\arctan \left(B_{z} / B_{y}\right) \sim 2 \pi / 5$. The reconnection field strength $\left|B_{z}\right|$ decreases as $\sqrt{1-z^{2} / L^{2}}$ along the current sheet, going to zero at the Y-lines at $z=$ $\pm L, x=0$. This magnetic configuration is force-free, so the density and gas pressure are initially set to be uniform at $\rho=\rho_{0}=1 / 2$ and $p=p_{0}$, which sets the ratio of plasma to magnetic pressure near the center of the current sheet at $\beta \equiv 8 \pi p /|\mathbf{B}|^{2} \sim 0.08$. The Alfvén speed used for normalization, $v_{A 0}=|\mathbf{B}| / \sqrt{4 \pi \rho}$, is measured near the center of the current sheet at $z=0, x \sim 0$.

Extrapolative, zero gradient, open boundary conditions are imposed in the $\hat{\mathbf{z}}$ and $\hat{\mathbf{x}}$ directions, while periodic boundary conditions are imposed in the $\hat{\mathbf{y}}$ direction. The computational mesh is adaptively refined in areas of high current magnitude. This gives a resolution ranging from 64 to 512 cells in the $\hat{\mathbf{y}}$, and $\hat{\mathbf{z}}$ directions $[-1.9 L, 1.9 L]$, and a resolution ranging from 32 to 256 cells in the $\hat{\mathbf{x}}$ direction $[-.95 L, .95 L]$. The current sheet is at the highest resolu- tion, and it is effectively one cell wide, so its thickness is $l=0.007 L$.

The simulation is run with a uniform background resistivity $\eta_{0}$. To initiate the reconnection, we impose a sphere of enhanced resistivity on the current sheet for the first $t v_{A 0} / L=0.6$ of the simulation. This resistive enhancement has the form $\eta=\eta_{0}\left(1+99 e^{-r^{2} / \delta^{2}}\right)$, for $r=$ $\sqrt{x^{2}+y^{2}+(z-2 L / 3)^{2}}<2 \delta$, with $\delta / L=0.087$. The Lundquist number of the background resistivity is $S_{\eta} \equiv$ $\delta v_{A 0} / \eta_{0}=5000$, while for the peak resistivity at the center of the reconnection region $S_{\eta}=50$. Note that Ugai (2007) have found that reconnection induced by locally enhanced resistivity is inhibited if $\delta<4 l$. As $\delta \sim 10 l$ here, the reconnection should not be inhibited by this mechanism.

\section{Results}

The effects of the magnetic reconnection event on the Ytype current sheet are shown in Figs. 1 and 2. Figure 1 shows a vector magnetogram view of the field in a plane perpendicular to the current sheet at $y=0$. Figure 1(a) is taken soon after the reconnection event is turned on. The guide field, shown by the greyscale, is enhanced in two small spots on either side of the reconnection event at $z=2 L / 3$. These spots are the cross sections of the two recently reconnected flux tubes flowing away from the reconnection site. The guide field is enhanced in these reconnected flux tubes because the reconnection component of the magnetic field has been annihilated, and the guide field must increase to make up for the lost magnetic pressure (see Hesse et al., 1996).

Figure 2(a) shows the fieldlines on either side of this current sheet just before the reconnection starts. Three topologically distinct sets of fieldlines are shown. One set 


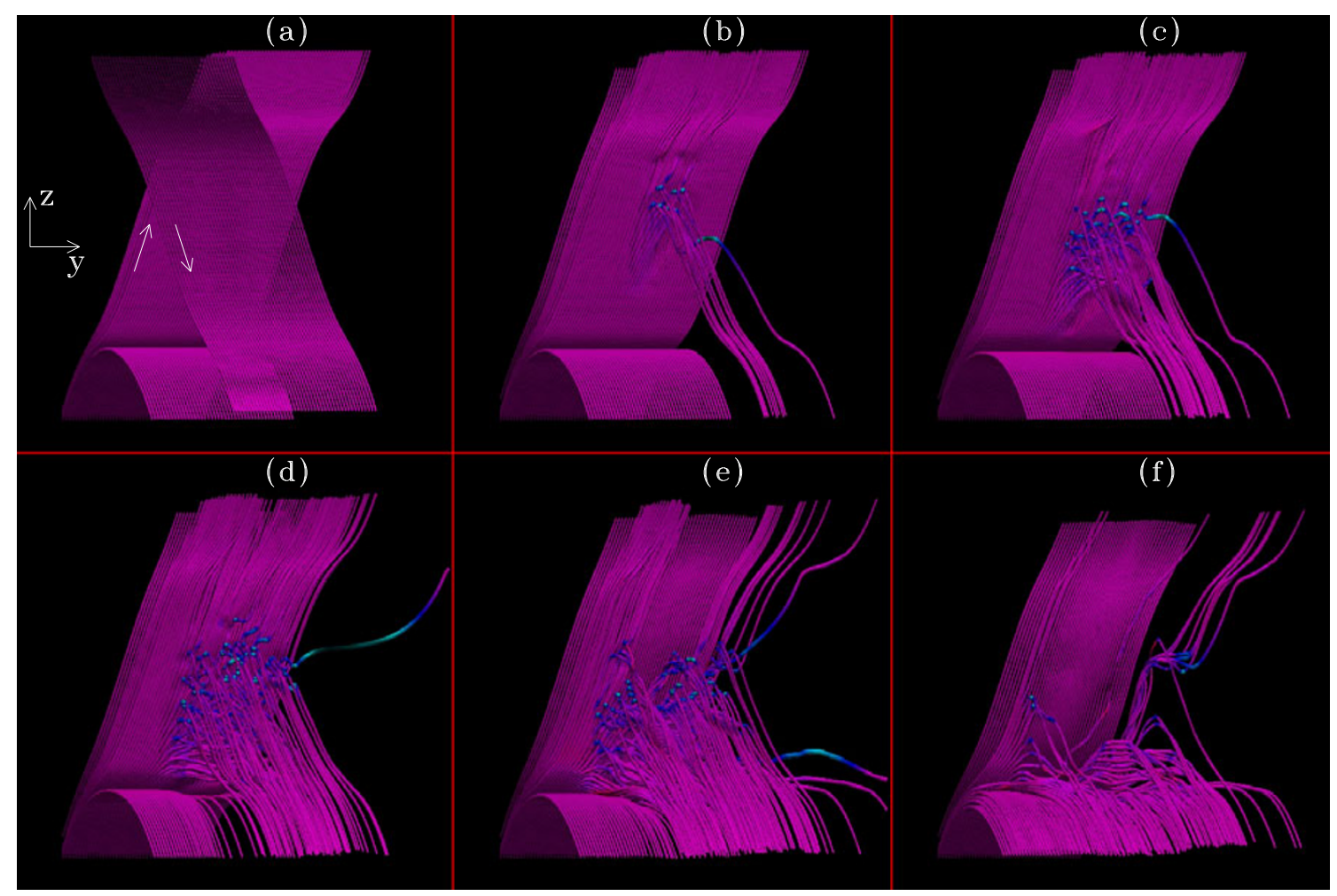

Fig. 2. Fieldlines of the reconnecting Y-type current sheet. The blue color shows where the electric current parallel to the magnetic field is strong. The panels show the simulation at times $t v_{A 0} / L=[0.0,1.3,2.1,2.8,3.4,3.9]$. Panel (a) shows fieldlines traced from both the front and back side of the current sheet, at the bottom boundary. The rest of the panels show only fieldlines traced from the back side of the current sheet, at the bottom boundary.

runs just behind the current sheet from the bottom to the top of the panel, while a second set runs in front of the current sheet from the top to the bottom of the panel, as marked by the arrows. The third set arches below the current sheet, running from the back of the simulation to the front, and coming close to the Y-line and the base of the current sheet at its apex. All three sets of fieldlines are canted gradually from left to right: this is the effect of the uniform guide field in the $\hat{\mathbf{y}}$ direction. The arched set of fieldlines represents the post-CME arcade loops while the two other sets of fieldlines represent the coronal current sheet fieldlines, which may still be connected to the CME above the second Y-line at the top of the simulation. For the remaining panels of Fig. 2, only the first and third sets of these fieldlines are traced, so that the second set, which lies in front of the current sheet, does not obscure the dynamics. Both of these sets of fieldlines are traced from the bottom boundary, behind the current sheet and arcade. Therefore, any section of a fieldline which appears in front of the current sheet above the arcade fieldlines is purely due to reconnection of frontside fieldlines with the back-side fieldlines.

The reconnection event was initiated very close to the upper Y-line, so the upflowing reconnected field quickly hits the Y-line, in Fig. 1(b). The corresponding upward retracting fieldlines are not shown in Fig. 2, since they are not connected to the lower boundary from which the fieldlines are traced. The upflow halts when it hits the upper Y-line and the arcade fieldlines lying above it. This is the equivalent of the upgoing fieldlines hitting and merging into the recently erupted CME, though this would only happen if the CME were erupting at a slower rate than the fieldlines retract.

The downflowing part of the reconnected flux is displayed in Fig. 2(b) as the fieldlines which trace upwards with all the field on the back side of the current sheet, but then suddenly take a hairpin turn and trace back down to the bottom boundary on the front side of the current sheet. The blue color of the fieldlines at these hairpin turns shows that the parallel electric current is enhanced and therefore reconnection is strong there (see, e.g., Schindler et al., 1988). The high placement of the initial reconnection region allows the cross section of the flux tube carrying this downflowing reconnected flux to fully take shape. Figures 1(b)-1(f) show how the cross section of the tube forms into an oblong shape, reminiscent of the coronal void observations, just as it did in the Harris sheet experiments of Linton and Longcope (2006). Note that the area of the downflowing flux tube cross section continues to grow as it absorbs more reconnected flux, even though the enhanced reconnection spot was turned off at $t v_{A 0} / L=0.6$, just before Fig. 1(b). Apparently the disturbance caused by the initial reconnection event spontaneously excites subsequent patchy reconnection events.

When this downflowing flux tube hits the Y-line and the arcade field below it, in Fig. 1(f), it decelerates and compresses the arcade field, in Fig. 1(g), and eventually joins the arcade, in Fig. 1(h). The reconnected flux which hits and joins the lower arcade is shown in Figs. 2(d)-2(f) as the fieldlines which now lie on top of the original arcade.

To illustrate the downflow and deceleration of the recon- 


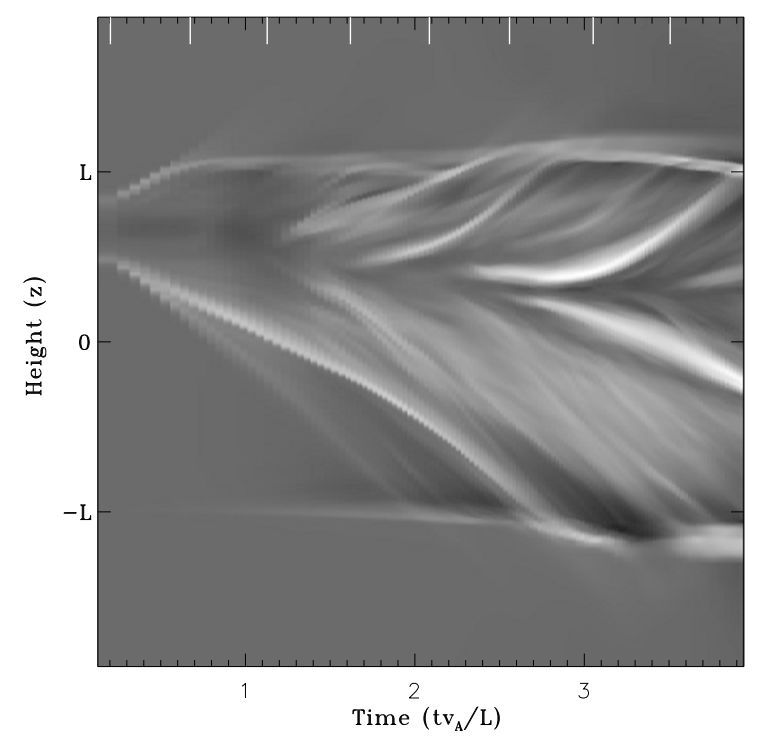

Fig. 3. Height-time plot of the guide field at $x=y=0$. The white tickmarks at the top mark the times of the vector plots of Fig. 1.

nected flux tubes, we show a height-time plot of the guide field along the $x=y=0$ line in Fig. 3. Here, the upward or downward propagating voids are displayed as diagonal white streaks of concentrated guide field. The first pair of up- and down-flowing streaks, starting at $t v_{A 0} / R=0$, are the tracks of the two initial reconnection voids. The upflowing track rapidly hits the upper Y-line and decelerates, while the downflowing track starts off at about $v_{A 0} / 2$, accelerates as it passes the current sheet midpoint, and then also rapidly decelerates as it hits the lower Y-line. Guidoni and Longcope (2007) suggest that a reconnected flux should accelerate near $z=0$ as seen here because the magnetic field strength, and thus the accelerating Lorentz force, peaks there.

After the reconnection is turned off at $t v_{A 0} / L=0.6$, no new tracks appear at the height of the initial reconnection site until about $t v_{A 0} / L=1.3$. Then patchy reconnection spontaneously sets in, as several reconnection outflow pairs appear in rapid sequence. This is supported by the more detailed views of Figs. 1 and 2. Figures 1(f)-1(h) show the corresponding newly reconnected flux tube cross sections flowing up and down at these times. Meanwhile, Figs. 2(c)-2(e) show the continual generation of newly reconnected fieldlines high up in the current sheet, well after the initial reconnection has been turned off and those reconnected fields have retracted. The source of this secondary patchy reconnection may be numerical resistivity due to the finite grid-scale of the simulation, in tandem with perturbations excited by the initial reconnection event, and will be explored in a future paper. Isobe et al. (2005) found a similar spontaneous patchy reconnection, but in their case it was due to an interchange instability, which cannot occur here. Interestingly, Fig. 1 also shows the reconnected fieldlines spreading in the $\hat{\mathbf{y}}$ direction along the current sheet, also in a patchy fashion, instead of remaining near the initial reconnection site. This is in contrast with the findings of Ugai et al. (2005) for a Harris current sheet without guide field. Their results show that initially localized reconnection does not expand significantly in this direction. From these preliminary results, it appears that the guide field can play an important role in expanding the reconnection region along the current sheet.

\section{Conclusions}

We have studied the effect of a brief, localized burst of reconnection in a Y-type current sheet with guide field. The up- and down-flowing flux this creates decelerates rapidly when it hits the Y-lines and the arcade fields beyond them. This gives strong support to the theory that the downflowing post-CME voids observed by TRACE, Yohkoh, and Hinode are in fact downflowing reconnected magnetic flux loops. This model gives a clear mechanism to explain why downflowing post-CME voids decelerate rapidly when they reach the post-flare arcades, as observed by Sheeley et al. (2004). We also find that the velocities of the voids change as they propagate through regions of different magnetic field strength in the current sheet. Finally, we find that the perturbation created by the initial burst of reconnection is sufficient to excite spontaneous patchy reconnection events in the current sheet.

Acknowledgments. This work was funded by NASA and ONR, with a grant of computer time from the DoD High Performance Computing program.

\section{References}

Asai, A., T. Yokoyama, M. Shimojo, and K. Shibata, Downflow motions associated with impulsive nonthermal emissions observed in the 2002 July 23 solar flare, ApJL, 605, 77-80, 2004.

Gallagher, P. T., B. R. Dennis, S. Krucker, R. A. Schwartz, and A. K. Tolbert, Rhessi and trace observations of the 21 April $2002 \times 1.5$ flare, Sol. Phys., 210, 341-356, 2002.

Green, J. M., Geometrical properties of three-dimensional reconnecting magnetic fields with nulls, in Solar and Stellar Magnetic Fields, edited by R. Lüst, 7 pp., North-Holland Publishing Co., Amsterdam, 1965.

Guidoni, S. and D. Longcope, Dynamics of post-reconnected thin flux tubes, Am. Astron. Soc. Meeting Abstracts, 210, \#93.27, 2007.

Hesse, M., J. Birn, M. M. Kuznetsova, and J. Dreher, A simple model of core field generation during plasmoid evolution, J. Geophys. Res., 101, 10797-10804, 1996.

Innes, D. E., D. E. McKenzie, and T. Wang, SUMER spectral observations of post-flare supra-arcade inflows, Sol. Phys., 217, 247-265, 2003.

Isobe, H., M. Takehiro, K. Shibata, and T. Yokoyama, Filamentary structure on the Sun from the magnetic Rayleigh-Taylor instability, Nature, 434, 478-481, 2005.

Linton, M. G. and D. W. Longcope, A model for patchy reconnection in three dimensions, ApJ, 642, 1177-1192, 2006.

McKenzie, D. E. and H. S. Hudson, X-ray observations of motions and structure above a solar flare arcade, ApJL, 519, 93-96, 1999.

Schindler, K., M. Hesse, and J. Birn, General magnetic reconnection, parallel electric fields, and helicity, J. Geophys. Res., 93, 5547-5557, 1988.

Sheeley, N. R., H. P. Warren, and Y.-M. Wang, The origin of postflare loops, ApJ, 616, 1224-1231, 2004.

Priest, E. and T. Forbes, Magnetic Reconnection, MHD Theory and Applications, Cambridge University Press, Cambridge, UK, 2000.

Ugai, M., Conditions for drastic evolution of magnetospheric current wedge, Phys. Plasmas, 14, 062902.1-062902.8, 2007.

Ugai, M., K. Kondoh, and T. Shimizu, Spontaneous fast reconnection model in three dimensions, Phys. Plasmas, 12, 042903.1-042903.7, 2005.

Welsch, B. T., C. R. DeVore, and S. K. Antiochos, Magnetic reconnection models of prominence formation, ApJ, 634, 1395-1404, 2005.

M. G. Linton (e-mail: linton@nrl.navy.mil), C. R. DeVore, and D. W. Longcope 